University of New Orleans

ScholarWorks@UNO

\title{
Performance of Chirp-Slope Keying with Joint Time-Frequency Detectors
}

Inigo X. Incer

University of New Orleans

Edit J. Kaminsky

University of New Orleans, ejbourge@uno.edu

Follow this and additional works at: https://scholarworks.uno.edu/ee_facpubs

Part of the Electrical and Electronics Commons

\section{Recommended Citation}

Incer, Inigo X. and Edit J. Kaminsky, "Performance of Chirp-Slope Keying with Joint Time-Frequency Detectors," Proceedings of the SPIE, Vol. 1677, Wireless Sensing and Processing II, 65770H, (2007). doi: $10.1117 / 12.717771$

This Conference Proceeding is brought to you for free and open access by the Department of Electrical Engineering at ScholarWorks@UNO. It has been accepted for inclusion in Electrical Engineering Faculty Publications by an authorized administrator of ScholarWorks@UNO. For more information, please contact scholarworks@uno.edu. 


\title{
Performance of Chirp-Slope Keying with Joint Time-Frequency Detectors
}

\author{
Iñigo X. Incer and Edit J. Kaminsky \\ Department of Electrical Engineering, University of New Orleans \\ New Orleans, LA 70148, U.S.A.
}

\begin{abstract}
This paper discusses recent studies on Chirp Slope Keying (CSK) as a scheme suitable for underwater communications and presents a new study on the performance of a time-frequency receiver in a Rayleigh fading environment. As expected, CSK proves to be a digital modulation scheme inferior in the AWGN channel as compared to traditional schemes but very promising in more detrimental channels present in underwater communications. In effect, while most schemes' performances decay abruptly with the addition of new disturbances, a time-frequency CSK receiver's performance deteriorates slowly with increasing Rayleigh fading. Intuition dictates that CSK will overpower other schemes as channel conditions continue to worsen. The receiver first uses the Wigner distribution to transform the incoming signal to the joint time-frequency domain and then computes the Radon transform to determine the binary digit received.
\end{abstract}

Keywords: Chirp modulation, time-frequency transform, Rayleigh fading, digital communications, underwater communications, underwater modem, underwater acoustical channel, acoustical communication.

\section{INTRODUCTION}

Underwater communications have been given increasing importance in recent years as a result of a rising number of commercial and military applications aimed at using the underwater channel. In effect, unmanned submersibles are increasingly used as the main means of operation in deep waters and have also started to replace humans in more accessible waters ${ }^{1}$. These are used for pollution control, offshore oil tasks, and reconnaissance, among others.

Communications underwater can be achieved using cables or a wireless system. Kaminsky and Barbu point out ${ }^{2}$ the impracticability of installing a wired communications system underwater. It is easy to note that not only would installation costs as such disallow this approach, but cable drag in mobile targets and exposure to the elements would do it as well. These reasons prevent further consideration to implementing a wired underwater communications system. Wireless systems, on the other hand, are appealing because of their ease of implementation and reduced cost of installation. These systems, however, cannot be implemented using electromagnetic waves, as traditionally done in the atmosphere, because the transmission of these waves only works well for the extra low frequency range. Considering the aforementioned, it is not surprising that the prevalent medium in underwater communications has been acoustics, as these waves enjoy superior propagation in this channel.

In order to quantify different schemes' performances in the Underwater Acoustic Channel (UWA), reliable models need to be developed. Some work is being performed in this area, but most studies have produced utterly complex models that hardly lend themselves to be applied in the evaluation of the communications schemes under consideration. Many authors have consequently recurred to simplified abstract models to take into account the possible disturbances introduced by the UWA channel ${ }^{3}$. The properties generally attributed to the UWA channel are Additive White Gaussian Noise (AWGN), multipath, Doppler spread, and phase shifts, among others. As mentioned ${ }^{2}$, the UWA channel may possibly be regarded as nature's most unforgiving transmission medium.

Chirp Slope Keying (CSK) is a digital chirp modulation scheme in which binary data are represented using chirps of increasing and decreasing frequencies versus time. In other words, an "up chirp" represents a ' 1 ' while a "down chirp" represents a "0." More bits can be transmitted simultaneously by increasing the number of frequency ranges or slopes of the frequency vs. time in the chirps. The nature of the chirp intrinsically has several desired characteristics in a

Wireless Sensing and Processing II, edited by Raghuveer M. Rao, Sohail A. Dianat, Michael D. Zoltowski, Proc. of SPIE Vol. 6577, 65770H, (2007) $\cdot 0277-786 \mathrm{X} / 07 / \$ 18 \cdot$ doi: $10.1117 / 12.717771$ 
communications system. In effect, chirps are spread spectrum, providing some imperviousness towards frequencyselective fading, detrimental to some modulation schemes such as Frequency Shift Keying (FSK). In addition, given that it is known beforehand that the possible transmitted signals have increasing and decreasing frequencies, it is possible to conceive a receiver architecture that does not require a phase synchronization mechanism. Note that phase defects can practically destroy some communication systems based on Phase Shift Keying (PSK).

This paper presents a review of two proposed receiver architectures for CSK: one based on time correlation and another one based on the second order time-frequency distributions of the chirps. The performances of the receivers have been studied in the AWGN channel ${ }^{2}$, and the time-correlation receiver has also been studied in a slow Rayleigh fading environment ${ }^{8}$. This paper summarizes published findings and introduces recent studies on the performance of the timefrequency receiver affected by slow Rayleigh fading.

The rest of this paper is organized as follows: The UWA channel is mathematically described with the presence of AWGN and Rayleigh fading in Section 2. Chirp Slope Keying is then presented as a digital modulation scheme in Section 3, followed in Section 4 by the CSK time-correlation and time-frequency receivers. The presentation of results for the operation and performance of CSK using the time-frequency receiver is given in Section 5. Concluding remarks and suggestions for further are given in Section 6.

\section{THE UNDERWATER ACOUSTICAL CHANNEL}

The UWA channel is generally modeled abstractly according to the undesired effects it introduces to the signals of interest. These effects include the ubiquitously present Gaussian noise, multipath, Doppler shifts, etc. This paper will cover only the first two.

\subsection{The AWGN Channel}

Many researchers model ambient noise underwater with the additive white Gaussian noise (AWGN) model. Using this model provides great mathematical tractability that sprouts from the properties of this noise. In effect, white Gaussian noise is a convenience that comes from the Central Limit theorem, and accurately describes many noise processes occurring in nature and in electronic systems. The resulting noise is described by $N_{o}$, the power spectral density of the AWGN process.

The signal-to-noise ratio (SNR) in the AWGN channel is defined as

$$
S N R, d B=10 \log _{10} \frac{W E_{b}}{\sigma^{2}},
$$

where $\sigma^{2}$ is the noise variance and $\mathrm{W}$ is the bandwidth of the chirp.

\subsection{Multipath and Slow Rayleigh Fading}

The signals that arrive at the receiver do not only come straight from the source. Messages sent to the receiver may bounce off on sea life or underwater boundaries or be refracted at different water depths. The information at the receiver then comes from multiple paths of various lengths and various phase delays, an effect called multipath.

Assume $r(t)$ is the received signal and $s(t)$, the sent signal. Following an approach similar to that in Proakis and Salehi ${ }^{4}$, it is assumed the message to be sent is a pure sinusoid

$$
s(t)=A \cos \left(2 \pi f_{c} t\right)
$$

The received signal, $r(t)$, can be expressed as 


$$
\begin{aligned}
& r(t)=A \sum_{n} \alpha_{n}(t) \cos \left\{2 \pi f_{c}\left[t-\tau_{n}(t)\right]\right\} \\
& =A \operatorname{Re}\left\{\sum_{n} \alpha_{n}(t) e^{-2 \pi f_{c} \tau_{n}(t)} e^{2 \pi f_{c} t}\right\} \\
& =\operatorname{Re}\left\{c(t) e^{2 \pi f_{c} t}\right\},
\end{aligned}
$$

where $\alpha_{n}(t)$ and $\tau_{n}(t)$ are, respectively, the attenuation factor and propagation delay corresponding to the $n^{\text {th }}$ propagation path; both are real signals. The signal

$$
\begin{aligned}
c(t) & =A \sum_{n} \alpha_{n}(t) e^{-2 \pi f_{c} \tau_{n}(t)} \\
& =A \sum_{n} \alpha_{n}(t) e^{-\phi_{n}(t)}
\end{aligned}
$$

can be considered the response of the channel to the complex exponential $e^{j 2 \pi f_{c} t}$.

Several parameters can be defined to quantify the response of the channel. Its spectral width, $B_{d}$, is called the Doppler frequency spread and quantifies the change in $c(t)$ with time. Another useful parameter is the multipath spread, $T_{m}$, defined as the time difference between the arrival of the first and last multipath components. The inverse of the multipath spread is called the coherence bandwidth, $B_{c b}$, which provides a measure of the frequency selectivity of the channel: that is, if $W$, the bandwidth of the message, is much smaller than $B_{c b}$, all frequency components of the signal are affected similarly; otherwise, they are affected differently.

Now, the multipath channel can be interpreted as a filter with a time-varying response $H(f ; t)$. Hence, $R(f)=H(f ; t) S(f)$, or

$$
r(t)=\int_{-\infty}^{\infty} H(f ; t) S(f) e^{-j 2 \pi f t} d f
$$

If the channel is not frequency selective, its frequency response can be considered constant. Thus, (4) becomes

$$
r(t)=c(t) \int_{-\infty}^{\infty} S(f) e^{-j 2 \pi f t} d f=c(t) s(t)
$$

Further insight and simplification are obtained by looking at the multipath spread of the channel. If the signal duration, $T$, is much larger than the multipath spread, then $c(t)$ becomes a constant that multiplies $s(t)$ during $T$. In those circumstances,

$$
c(t)=c_{r}(t)+j c_{i}(t)
$$

where $c_{r}(t)$ and $c_{i}(t)$ are complex-valued Gaussian random processes. A more insightful way of writing (6) is as follows

$$
c(t)=\alpha(t) e^{j \phi(t)}
$$

It can easily be shown that $\alpha(t)$ and $\phi(t)$ are Rayleigh and uniform random processes, respectively. In effect, given that different multipath components travel over different distances, their phase probably changes equally between 0 and $2 \pi$, 
while the multiplicative constant does not change as drastically. In addition, the multiplicative constant will sometimes increase the signal energy at the receiver when signals undergoing different paths add constructively but will reduce it when they add destructively, an effect called signal fading.

We define the SNR as

$$
S N R=10 \log _{10} \frac{E_{b}}{N_{o}} 2 \sigma^{2},
$$

where $2 \sigma^{2}$ is the energy contributed by the multipath components and $N_{0}$ is the power spectral density of the AWGN.

\section{CHIRP SLOPE KEYING}

Binary chirp slope keying (CSK) is a digital modulation scheme in which two chirps of increasing or decreasing frequencies are used to represent binary data. Chirps were apparently suggested as a modulation scheme for communications systems by Winkler ${ }^{5}$ and studied by Berni ${ }^{6}$ in the 1970's but had been in use in radars since the 1930 's. The idea of modulating digital data using chirps has risen in interest in recent years.

The basic signals of CSK or what Berni ${ }^{6}$ calls Linear Frequency Sweeping (LFS) are

$$
s_{u}(t)=A \cos \left[2 \pi t\left(f_{\min }+\frac{f_{\max }-f_{\min }}{T} \frac{t}{2}\right)\right]
$$

and

$$
s_{d}(t)=A \cos \left[2 \pi t\left(f_{\max }+\frac{f_{\min }-f_{\max }}{T} \frac{t}{2}\right)\right]
$$

Note the frequencies of the chirps vary over time as

$$
f_{u}(t)=\frac{1}{2 \pi} \frac{d}{d t}\left[2 \pi t\left(f_{\min }+\frac{f_{\max }-f_{\min }}{T} \frac{t}{2}\right)\right]=f_{\min }+\frac{f_{\max }-f_{\min }}{T} t
$$

and

$$
f_{d}(t)=f_{\max }+\frac{f_{\min }-f_{\max }}{T} t .
$$

In the equations above, $T$ is the signal duration per bit. The energy of the chirps can be computed by integrating the square of the magnitude of the chirps. These integrals are computed numerically, given the presence of Fresnel integrals. It is found the energy of a single bit is approximately 0.5 and the crosscorrelation between the up and down chirps for $\tau$ $=0$ is small.

\section{CSK RECEIVER STRUCTURES}

This section presents two receiver architectures that have been proposed for CSK: the typical receiver based on time correlation and a recently proposed receiver based on the detection of the time-frequency distribution of the incoming signal $^{2}$. 


\subsection{The Time Correlator}

Receivers based on time correlation are very common in literature as a result of their closeness to the ideal receiver -the matched filter- for the AWGN channel. This ideal receiver aims to maximize the signal to noise ratio (SNR) in the signal $r(t)$ sensed at the receiver. This signal has the form

$$
r(t)=s(t)+n(t)
$$

where $s(t)$ is the sent signal, and $n(t)$ is AWGN with $N_{o}$ power per absolute frequency.

After a concise derivation, it is found that the impulse response of the receiver to achieve the desired maximization in the SNR is given by the expression stated below ${ }^{7}$. A filter with this impulse response is called a Matched Filter.

$$
h(t)=K s^{*}\left(t_{0}-t\right)
$$

where $\mathrm{K}$ is a constant, and $\mathrm{t}_{\mathrm{o}}$ is the time when maximization is desired.

A receiver proposed ${ }^{8}$ multiplies the incoming chirp signals by an up chirp. In the absence of noise, integration of the incoming signal would produce 0.5 if a " 1 " is sent or a 0 if a " 0 " is sent. Hence, the result of the integration is compared to 0.25 , chosen empirically by assuming equal probability for a 1 and a 0 . A block diagram of this receiver is given below

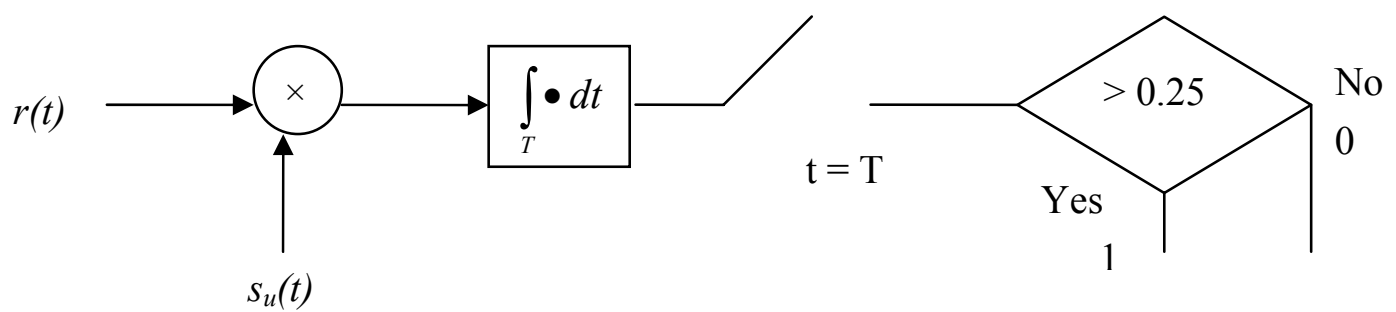

Fig. 1. Block diagram for the time-correlation receiver for CSK

\subsection{The Time-Frequency Receiver}

Another type of receiver structure viable for CSK demodulation is one based on analysis of the power spectrum of the received signal. This receiver was introduced in ${ }^{2}$. Consider the power spectra of the up and down chirps. 


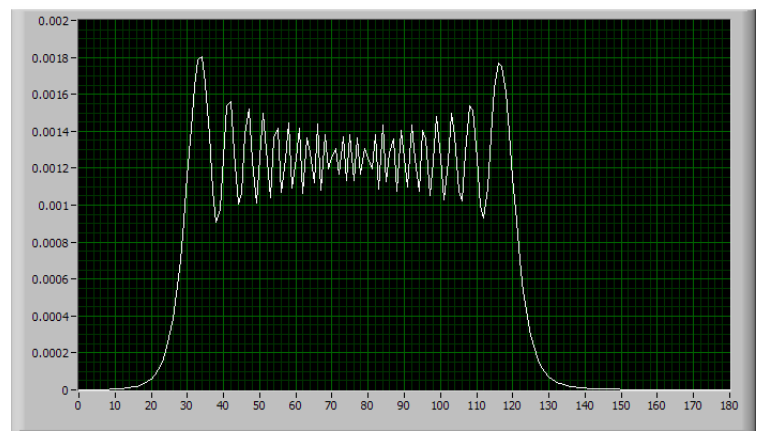

Fig. 2. Frequency spectrum of an up-chirp.

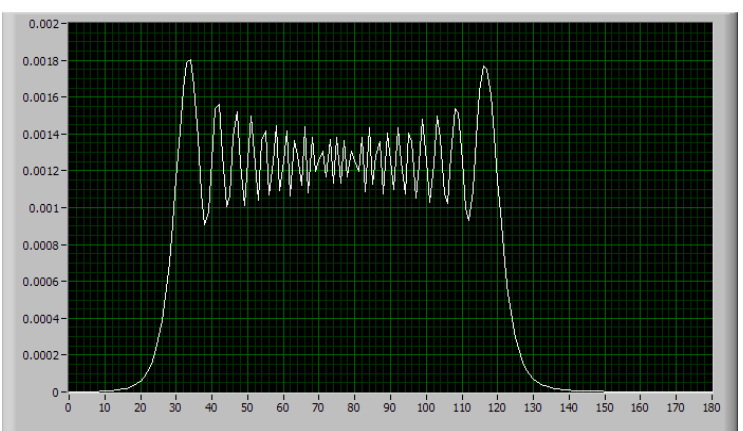

Fig. 3. Frequency spectrum of a down-chirp.

Figures 2 and 3 show the power spectra of up and down chirps using $25 \mathrm{~Hz}$ and $125 \mathrm{~Hz}$ for the minimum and maximum frequencies, respectively, at a $512 \mathrm{~Hz}$ sampling rate. It may be noticed the spectra are exactly the same, but intuition tells that the frequency content of the received signals varies in a linear fashion with time. The way to extract this varying content is by using joint time-frequency distributions. The idea is to produce a distribution $P(t, f)$, such that the energy of the signal is given by

$$
E=\iint P(t, f) d f d t
$$

Cohen ${ }^{9}$ shows an infinite number of distributions can be produced that fulfill the property above; these are of the form given in (15)

$$
P(t, \omega)=\frac{1}{4 \pi^{2}} \iiint e^{-j \theta t-j \tau \omega+j \theta u} \phi(\theta, \tau) s^{*}\left(u-\frac{1}{2} \tau\right) s\left(u+\frac{1}{2} \tau\right) d u d \tau d \theta
$$

where $\phi$ is called the kernel.

A well known and often used distribution is obtained when the kernel is equal to 1 . It is called the Wigner-Ville distribution, and it fits the purposes of the time-frequency receiver proposed for CSK because it provides a very accurate resolution for continuous signals. The Wigner-Ville distributions of up and down chirps are shown in Figures 4 and 5.

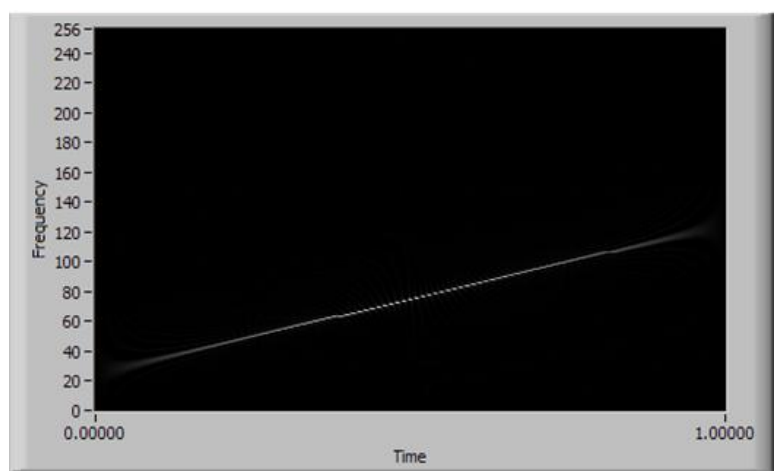

Fig. 4. Wigner-Ville distribution of an up-chirp

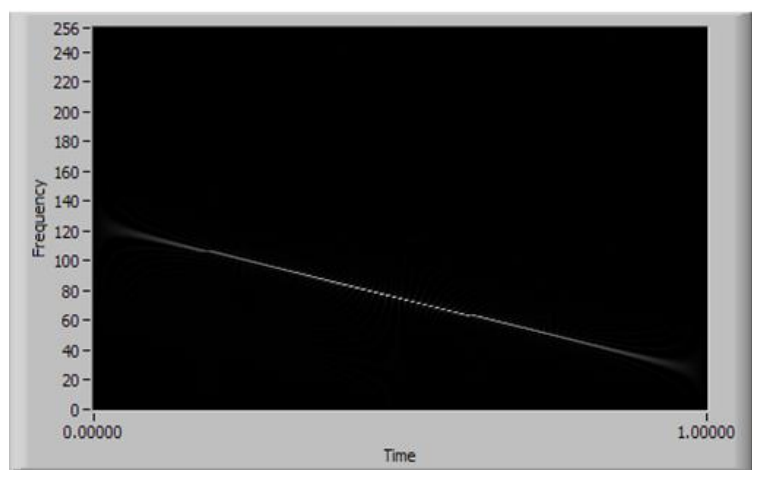

Fig. 5. Wigner-Ville distribution of a down-chirp 
It is clear from Figures 4 and 5 that chirps with different slopes can be easily differentiated using time-frequency distributions. Indeed, the distributions fulfill expectations since the chirps they represent change their frequencies from $25 \mathrm{~Hz}$ to $125 \mathrm{~Hz}$ linearly in the $1 \mathrm{~s}$ period. In order to complete the receiver, however, a mechanism to detect a line and its slope has to be devised, and this can be performed in many ways. Edge detection filters, for example, can be used for pixel linking; and linear regression, for parameter estimation, but this approach, as most alternatives, suffers from poor performance in noisy environments ${ }^{10}$. The Radon transform, on the other hand, fits the requirements ideally.

Let $g(x, y)$ be a two dimensional function. Its normal Radon transform, $g(\rho, \theta)$, is defined as

$$
\stackrel{\vee}{g}(\rho, \theta)=\iint g(x, y) \delta(\rho-x \cos \theta-y \cos \theta) d x d y
$$

It can readily be shown that given a line $g(x, y)=\delta\left(\rho^{*}-x \cos \theta^{*}-y \cos \theta^{*}\right)$, its radon transform is

$$
g(\rho, \theta)=\left\{\begin{array}{l}
0, \quad \rho \neq \rho^{*} \text { or } s \neq s^{*} \\
\int_{-\infty}^{\infty} \delta(0) d s, \quad \rho=\rho^{*} \text { and } s \neq s^{*},
\end{array}\right.
$$

showing that a line is easily recognizable in the transform domain. The Radon transform of the Wigner-Ville distributions of the up and down chirps are shown in figures 6 and 7, respectively (the center of the image is taken as the origin).

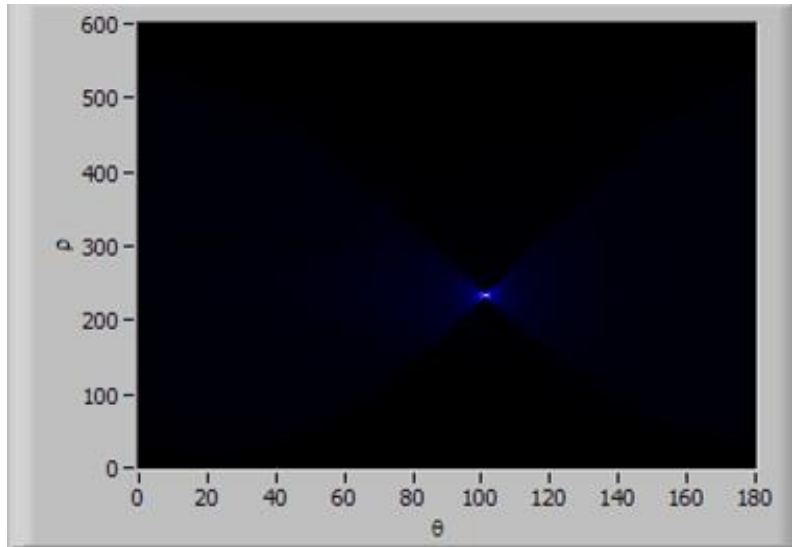

Fig. 6. Radon transform of the Wigner-Ville distribution corresponding to an up-chirp. Angle $\theta$ in degrees.

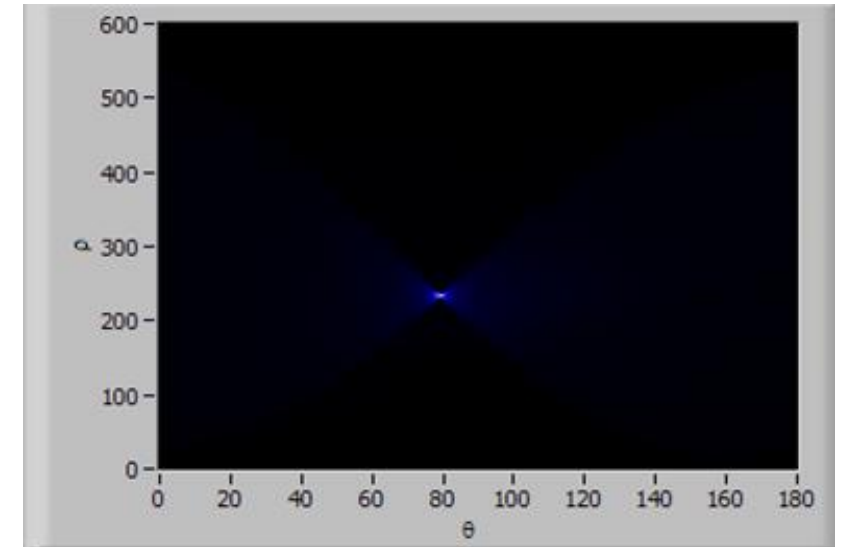

Fig. 7. Radon transform of the Wigner-Ville distribution corresponding to a down-chirp. Angle $\theta$ in degrees.

We easily see from Figures 6 and 7 that distinction between the two transmissions can be made using the Wigner-Ville distribution followed by the Radon transform. The angles at which maximum reception occur for up and down chirps are 101 and 79 degrees, respectively. The results of the radon transform at angles larger than 90 degrees (midpoint between 79 and 101) are compared to those at angles smaller than 90 degrees, and whichever is larger determines the bit understood by the receiver. In other words, if the transform is greater at an angle larger than 90 degrees, a " 1 " is assumed as sent; otherwise, a " 0 " is assumed.

\section{RESULTS}

The results for the probability of error (PE) of BPSK are initially given for later comparison with the results obtained for the CSK time-frequency receiver. BPSK has closed form expressions for the PE vs. SNR for the AWGN and slow Rayleigh channels. The PE in the AWGN is given by 


$$
P_{2}=Q\left(\sqrt{\frac{2 E_{b}}{N_{o}}}\right),
$$

where

$$
Q(x)=\frac{1}{2} \operatorname{erfc}\left(\frac{x}{\sqrt{2}}\right) .
$$

The expression for the Bit Error Rate (BER) of BPSK in the slow Rayleigh fading environment is ${ }^{4}$ :

$$
B E R=\frac{1}{2}\left[1-\sqrt{\frac{S N R}{1+S N R}}\right] .
$$

Note the Rayleigh multiplicative noise adds (or subtracts) energy from the signal. SNR in (19) is, again, defined as

$$
S N R=\frac{E_{b}}{N_{o}} E\left[\alpha^{2}\right]=\frac{E_{b}}{N_{o}} 2 \sigma^{2},
$$

where $2 \sigma^{2}$ is the energy contributed by the multipath components and $N_{0}$ is the power spectral density of the AWGN

Matlab $^{1}$ simulations were performed ${ }^{8}$ to study the performance of the time-correlation receiver for CSK. Figure 3 in Kaminsky and Simanjuntak ${ }^{8}$ summarizes the findings for the AWGN channel and the slow Rayleigh fading channel using different values of sigma for the multiplicative Rayleigh noise.

Here, we studied the performance of the time-frequency receiver (Wigner distribution followed by the Radon transform) for CSK receiver was studied in the AWGN and slow Rayleigh fading environments by running simulations in LabVIEW $^{2}$. The results are summarized in Fig. 8 where we also show simulation results for CSK in AWGN and the performance of PSK in the same Gaussian channel.

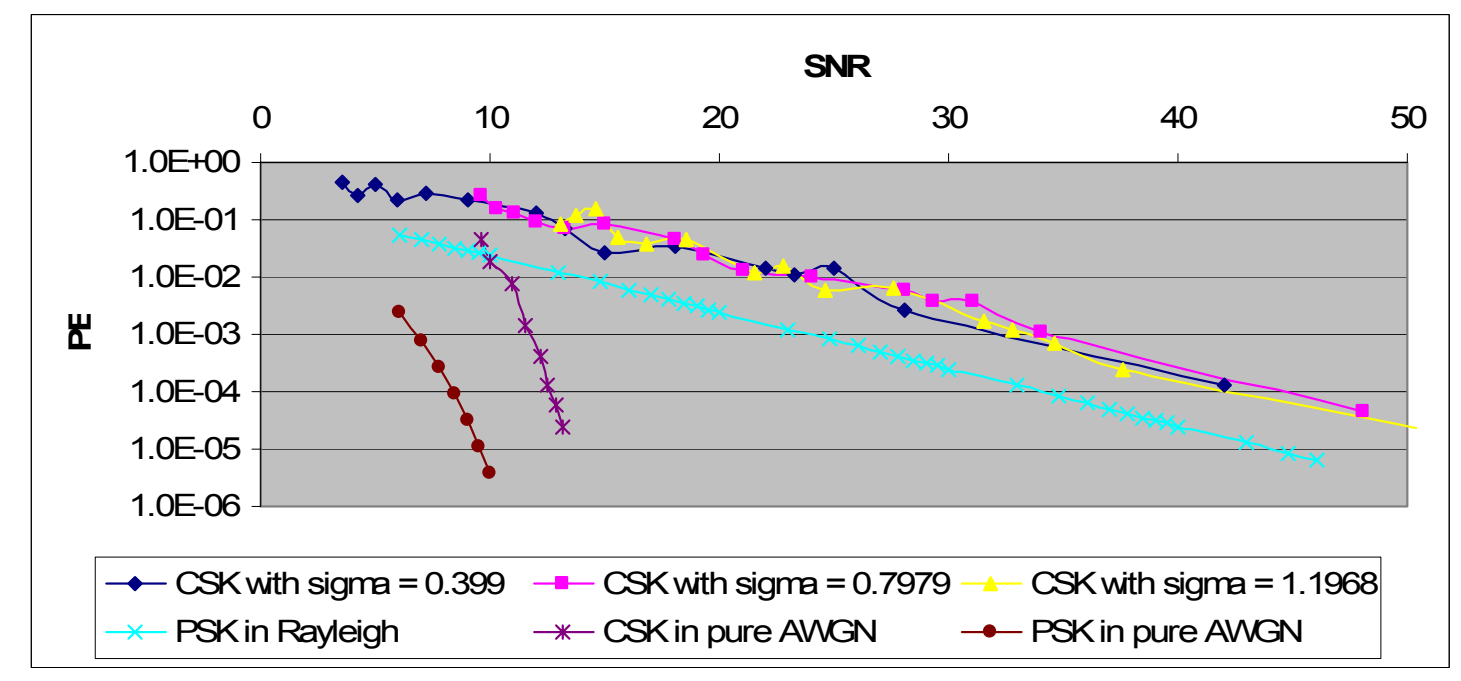

Fig. 8. CSK performance (PE vs. SNR) in AWGN and slow Rayleigh fading channels using a time-frequency receiver.

\footnotetext{
${ }^{1}$ Matlab $^{\mathrm{tm}}$ is a registered trademark of The MathWorks, Inc.

${ }^{2} \mathrm{LabVIEW}^{\mathrm{tm}}$ is a registered trademark of National Instruments Co.
} 
Notice that simulation of CSK indicates a degradation that is almost a straight line in the PE vs. SNR (dB) plane. Moreover, there is only negligible difference in performance as the Rayleigh parameter is changed.

\section{CONCLUSIONS}

The performances for the various schemes are given in Figures 8 and 9. Although BPSK defeats CSK (both Correlator and time-frequency receiver) in both the AWGN and the slow Rayleigh fading channels -for most reasonable values of SNR-, it is expected that when other deleterious channel effects, such as Doppler and phase delays, are incorporated, CSK will perform better than PSK.

The comparison of the two receivers for CSK, however, showed that the time-frequency receiver performance is worse than the time-correlation receiver in the AWGN. This is to be expected because the time-correlation receiver is closer to the ideal receiver in AWGN than the time-frequency receiver. It is worthy to note, however, the two receivers seem to converge for higher SNR values. In the Rayleigh fading environment, however, the time-frequency receiver shows sturdy performance as compared to the time-correlation receiver. The performance of the time-frequency receiver does not seem to depend on particular values of the sigma parameter of the multiplicative Rayleigh, while the time-correlation receiver does. This is clear from Fig 8, which shows the time-correlation receiver's performance decays abruptly for higher values of the Rayleigh sigma.

These results still don't provide a clear advantage to CSK over traditional schemes. However, the addition of more imperfections is thought to show this advantage in favor of the time-frequency receiver in CSK. In effect, as already mentioned, the time-frequency receiver can easily be conceived as not needing a phase recovery mechanism even in the presence of phase offsets and time delays since the two lines that would appear in the time-frequency distributions of the incoming signals could be differentiated. This is opposed to the effects these channel conditions would have on PSK, sensitive to these types of imperfections.

Another area worthy of serious research is the modeling of the UWA channel so that simulations may bring results closer to those that would be obtained in the physical environment.

\section{REFERENCES}

1. M. Stojanovic, Underwater Acoustic Communications, IEEE Electro International, pp. 435-440, June 1995.

2. E. Kaminsky and M. Barbu, Receiver structures for underwater acoustical communications using chirp slope keying, Proc. SPIE 6201 (37), 2006.

3. A. B. Baggeroer, Acoustic Telemetry -An Overview, IEEE Journal of Oceanic Engineering OE-9 (4), pp. 229$235,1984$.

4. J. G. Proakis and M. Salehi, Fundamentals of Communications Systems, Pearson Education, Inc., Upper Saddle River, New Jersey, pp. 623-635, 2005.

5. M. R. Winkler, Chirp Signals for Communications, WESCON Convention Record 14 (2), 1962.

6. A. J. Berni and W. D. Greeg. On the Utility of Chirp Modulation for Digital Signaling, IEEE Trans. on Commun. 21 (6), pp. 748-751, 1973.

7. X. R. Li, Probability, Random Signals, and Statistics, CRC Press, Boca Raton, Florida, pp. $415-422,1999$.

8. E. J. Kaminsky and L. Simanjuntak, Chirp Slope Keying For Underwater Communications, Proc. SPIE 5778, pp. 894-905, 2005.

9. L. Cohen, Time-frequency distributions -A review, Proc. IEEE 77 (7), pp. 941-981, 1989.

10. P. Toft, The Radon Transform - Theory and Implementation, Ph.D. thesis, pp. 3 - 36, 1996, < http://petertoft.dk/PhD/>, Accessed March 5, 2007. 